\section{A Simple Technique for Image- Guided Excision of Non-Palpable Breast Lesions without Needle Localisation in a Low-Resource \\ Environment - a Preliminary Report of 10 Patients}

Jameel Ali ${ }^{1}$, Fidel Shilandra Rampersad ${ }^{1}$, Brittney Lutchman ${ }^{1}$, Adam Hanna ${ }^{1}$, Jason Rattan ${ }^{1}$, Johnathan Jarvis $^{1}$, Malini Ramnarine ${ }^{1}$, Nicholas Babootee ${ }^{1}$, Richard Spence ${ }^{1}$, David Malcolm Milne ${ }^{1}$, Anna Radha Rampersad $^{1}$

${ }^{1}$ St. James Medical Complex, Western Main Rd, St James, Trinidad and Tobago

\section{Corresponding Author:}

Professor Jameel Ali

St James Medical Complex 112,

Western Main Rd,

St James,

Trinidad and Tobago

Email: jameel.ali@unityhealth.to

\section{DOI: doi.org/10.48107/CMJ2021.04.006}

Copyright: This is an open access article under the terms of the Creative Commons Attribution License which permits use, distribution and reproduction in any medium, provided the original work is properly cited.

(C)2021 The Authors. Caribbean Medical Journal published by Trinidad \& Tobago Medical Association

\section{ABSTRACT}

Focus on early diagnosis of non-palpable breast cancers has frequently led to expensive interventions that are more challenging in low-resource environments. We examined the use of a simple, inexpensive technique more suited for these environments, presenting preliminary data on its applicability.

In 10 consecutive patients with radiologically suspicious non-palpable breast lesions, we used skin markers and breast reference points generated through ultrasound images and clinical orientation to guide location of surgical incisions for excising these lesions in the operating room. This resulted in accurate localisation and complete excision of these lesions without the need for more sophisticated, expensive interventions, making this technique potentially more applicable in low-resource environments, such as ours.

Keywords: Non-palpable breast lesions; treatment of non-palpable breast masses; ultrasound marking of nonpalpable breast lesions.

\section{BACKGROUND}

Breast cancer is among the leading causes of cancer related deaths in women internationally and especially in the Caribbean. ${ }^{1,2}$ The major determinant of outcome is early diagnosis ${ }^{3}$ with the emphasis on screening strategies and increased awareness. This approach has resulted not only in the desired earlier diagnosis but the identification of 'occult' smaller frequently non-palpable lesions that are more challenging to identify, biopsy and treat definitively. In most centres these lesions are biopsied or excised by utilising image guided wire localisation. ${ }^{4}$ More sophisticated techniques utilising dyes, isotopes etc. have been described and successfully applied $^{5-7}$ in treating these non-palpable lesions of the breast.

In Trinidad and Tobago, the largest breast cancer referral centre is publicly funded and in many instances the institution is unable to fully provide all resources necessary for optimum care in a timely fashion making it necessary to access privately funded institutions. Many of the patients attending the public institutions do not have 
private insurance and cannot afford to pay for the sophisticated techniques that are frequently employed in providing that care in private institutions. Although these interventions may be available in the public setting they may not be provided in a timely manner. ${ }^{8}$ To minimize cost and yet provide appropriate treatment for this patient population we pursued a simple technique, not requiring wire localisation or other sophisticated modalities as described in the literature (such as isotope injection, dye injection, seed placement, intraoperative ultrasound. ${ }^{5-7}$ We proceeded to analyse the outcome as part of a project to determine if this can be successfully applied in our low-resource environment.

\section{The Technique}

The non-palpable lesion is usually identified on a previous ultrasound or mammographic study and, if it is considered suspicious enough to warrant excision, the patient is invited to participate in the study. If she chooses to participate in the study, the rationale, potential risks, benefits and complications are explained followed by signed witnessed informed consent as stipulated by the Research Ethics Committee of the North-West Regional Health Authority. The radiologist also reviews the consent form followed by similarly signed informed consent about the radiologic procedure. The radiologist then identifies and describes the precise location of the lesion using reference points such as depth from the skin, distance from the nipple and the proper vector (position referring to the numbers on the clock face e.g., the three o'clock position). This information is contained in the radiologist report prepared on the day of the placement of the marker which is conducted on the day before the surgery. The two perpendicular lines of the marker intersect directly over the lesion identified on ultrasound. The marked area is covered with a secure dressing. The surgery is conducted under general anaesthesia and the previously obtained ultrasound image and report are available in the operating room. The radiologist and surgeon discuss the report prior to the surgery and the surgeon makes the incision through the centre of the cross made by the skin marker. A copy of the radiologist report and a picture of the skin marker are shown as follows (see Figure 1 for picture of the marker below). A sample radiology report is as follows:
'Ultrasound confirms a solid well-circumscribed mass in the left five to six o'clock position, located $3 \mathrm{~cm}$ from the nipple and $0.4 \mathrm{~cm}$ deep to the skin. The lesion measures $1.1 \times 0.6 \mathrm{~cm}$. The position on the skin immediately overlying the lesion is marked by the intersection of the two lines.'

We used this combination of the radiologist report and the skin marker to guide us in excision of specimen which involves an incision down to the pectoral fascia through the intersection of the two lines on the skin.

The presenting features of the patient-age, all femalescharacteristics of the lesion (the pathology, size of the lesion, size of the excised specimen) from 10 consecutive patients who agreed to participate are shown in the following table with the size of the excised specimen in parenthesis:

As shown in this table, only two of the 10 specimens proved to be malignant, but all 10 were considered suspicious enough on imaging to warrant excision.

\section{Figure 1. Skin markers on breast with intersecting $\mathbf{2} \mathrm{cm}$ above the areola.}

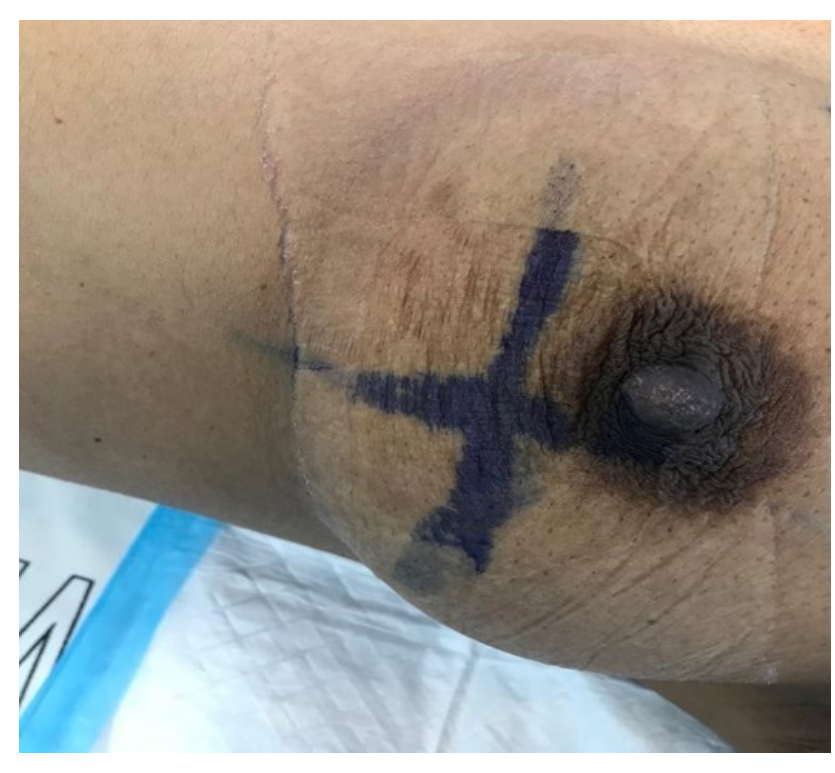


Table 1: Characteristics of the patients and the excised lesions

\begin{tabular}{|c|c|c|}
\hline Patient \# & Age & Histology/Size of lesion; size of specimen in brackets \\
\hline 1. & 36 & Fibroadenoma;2.0x1.8x1.6 cm; $(2.5 \times 2 \times 3 \mathrm{~cm})$ \\
\hline 2. & 41 & $\begin{array}{l}\text { Intraductal papilloma, apocrine metaplasia and benign ducto-lobular units; } 0.3 \times 0.2 \times 1 \\
\mathrm{~cm}(2.5 \times 1.5 \times 2 \mathrm{~cm})\end{array}$ \\
\hline 3. & 73 & Invasive Carcinoma with lobular features, grade $2 ; 3.7 \times 1.2 \times 1.5 \mathrm{~cm} ;(5 \times 2 \times 2.5 \mathrm{~cm})$ \\
\hline 4. & 46 & $\begin{array}{l}\text { Breast parenchyma with mild chronic inflammation, fibrocystic changes, apocrine met- } \\
\text { aplasia and dense stromal fibrosis; size of excised specimen is } 6.8 \times 4.5 \times 5.3 \mathrm{~cm}\end{array}$ \\
\hline 5. & 46 & $\begin{array}{l}\text { Fibroadenosis. Multiple areas of adenosis, stromal fibrosis, and dilated ducts lined with } \\
\text { apocrine metaplastic glandular cells and blunt duct adenosis. Atypical cells line terminal } \\
\text { ducts with associated microcalcification; }(2.5 \times 3.5 \times 1.5 \mathrm{~cm})\end{array}$ \\
\hline 6. & 56 & $\begin{array}{l}\text { Well circumscribed cyst lined with double layer of cuboidal cells along with surrounding } \\
\text { stromal inflammatory response; } 1.5 \times 1 \times 0.8 \mathrm{~cm} ;(2 \times 2 \times 1.5 \mathrm{~cm})\end{array}$ \\
\hline 7. & 33 & Fibroadenoma; $2 \times 2 \times 1 \mathrm{~cm} ;(2.5 \times 2 \times 2.5 \mathrm{~cm}$ ?) \\
\hline 8. & 63 & $\begin{array}{l}\text { Invasive Carcinoma grade } 2 \text { which has lobular pattern. } 1.25 \times 1.0 \times 1.5 \mathrm{~cm} ;(2.0 \times 2.0 \times 1.4 \\
\mathrm{cm})\end{array}$ \\
\hline 9. & 76 & $\begin{array}{l}\text { Previous WLE for grade } 2 \text { ductal invasive carcinoma. Imaging which prompted the exci- } \\
\text { sion showed BIRADS } 4 \text { microcalcifications on mammography visible also on ultrasound. } \\
\text { Sample excised is ( } 7.4 \times 4.5 \times 2.5 \mathrm{~cm} \text { ) consisting of benign breast parenchyma with micro- } \\
\text { calcifications and stromal fibrosis from previous surgery. No malignancy seen. }\end{array}$ \\
\hline 10. & 48 & $\begin{array}{l}\text { Previous Left WLE. BIRADS } 4 \text { lesion on follow-up ultrasound - scar tissue vs malignan- } \\
\text { cy. Histology shows fibrous tissue and chemoradiotherapy effects modifying acini but } \\
\text { no evidence of residual carcinoma. Areas of pleomorphic calcification were present; } \\
(3 \times 2 \times 1.5 \mathrm{~cm})\end{array}$ \\
\hline
\end{tabular}

A postoperative ultrasound was performed 2-6 weeks after the excision at the convenience of the patient to determine if the lesion which prompted the excision had been totally removed.

In all 10 patients the previously noted lesion was no longer present on the follow-up ultrasound or mammogram in the one patient with microcalcification.

\section{DISCUSSION}

Our aim in this project was to test the feasibility of resecting non-palpable breast lesions using skin markers and ultrasound with precise radiologic reporting as a guide for the surgical procedure, particularly location of the incisions.

The size of the lesions reported on the table above may suggest that some of these lesions may not be truly nonpalpable, but palpability is related not only to size but also depth of the lesion and the size of the breast. Also, the size of the specimen removed and reported by the pathologist was frequently larger than the size reported on the preoperative ultrasound because of the desire to obtain clear margins in the excised specimens. Patient \#9 had a previous wide local excision and postoperative 
radiotherapy for invasive ductal carcinoma, then developed a wide area of BIRADS (Breast Imaging Reporting and Database Systems) 4 microcalcification on follow-up mammography with no palpable lesion several years later. BIRADS is a system of classifying the radiologic abnormality to guide treatment based on the degree of suspicion of malignancy with a grading of BIRADS 0 to 6 . BIRADS 4 represents up to $40 \%$ risk of malignancy warranting excision or at least biopsy. ${ }^{9}$ The microcalcifications were also visible on ultrasound, so our skin marking ultrasound technique was used to guide the incision location. Radiography of the very wide $(7.4 \mathrm{~cm})$ excised area showed that all the microcalcifications had been excised and no malignancy was identified.

We were able to achieve our goal in all 10 of our patients of accurately identifying and excising the targeted lesions arriving at a satisfactory and successful patient outcome without requiring the more expensive localisation wire or other more sophisticated devices as described in the literature.

This procedure requires an experienced skilled dedicated radiologist as we were fortunate to have available locally and a close working team relationship with the surgeon.

The radiologist-FR - had his DM Radiology training at the University of the West Indies, FRCR from the UK, Interventional Radiology Fellowship in Indonesia, and wide clinical experience in interventional radiology with a major focus in Breast Radiology over the last eight years. The consultant Surgeon-JA-is Professor of Surgery, University of Toronto, and Head of Breast Surgical Oncology at St. James Medical Complex in Trinidad for the past five years with a Postgraduate Fellowship in Surgical Breast Oncology at St Michael's Hospital, University of Toronto.

We plan to gain further experience with this technique with the aim of applying it more widely for our patients especially as the resource requirement is minimal and easily achievable in our environment. Earlier postoperative imaging and intraoperative imaging, as well as quick frozen section by the pathology department, could also enhance the effectiveness of this technique in our setting.

\section{LIMITATIONS}

We embarked on this study primarily to determine if a simple technique which avoids expensive interventions could yield results that are helpful in the clinical management of suspicious non-palpable breast lesions with potential application in low-resource environments. Although we were fortunate to obtain excellent results in that we were able to treat all these patients effectively and definitively in our setting with a simple inexpensive technique, caution must be exercised in applying this technique generally for several reasons. Although our results are encouraging, they can only be considered preliminary, first of all, because our sample was very small, warranting more study. We plan to analyse more patients with possibly a control group having the standard proven but more expensive treatment. Secondly, this technique requires the services of a dedicated, specially trained, interventional radiologist and experienced surgeon with good team relationships, as we were fortunate to have. It is obvious that we would not be discussing application of these techniques if our public institutions are better equipped to meet our patients' needs-a strong political will is needed to effect this type of change. A vailability of intraoperative ultrasound and immediate postoperative imaging of specimens and quick pathologic frozen section would enhance effectiveness of treatment-this could be obtained in many relatively lowresource environments. We conducted imaging follow-up 2-6 weeks following surgery, which is not ideal. With some extra effort, immediate postoperative imaging may be possible, even in our environment, and likely result in more reliable and predictably better outcome, notwithstanding our overall good result in this study. The pendulous breast with mobile lesions would probably be more challenging and be fraught with more inaccuracy. Although we did not use these as a criteria for selection of cases, our cases largely did not fall in that category thus possibly yielding a better outcome. This should be considered if undertaking our simplified technique.

In pursuing the techniques described in our study, we were mindful of past experience using skin marking and orthogonal localisation techniques which were abandoned because of increased rates of re-excision and larger volumes of tissue excised. The question we attempted to address in our study was whether we could use our technique that is more fitting for a low-resource environment and yet avoid re-excisions and excessive tissue removal. Fortunately, in our study, though limited 
by small numbers, we were able to avoid these drawbacks to some extent because of our patient selection-small, non-pendulous breasts with lesions that were not very mobile. This should probably be considered by others in low-resource environments who wish to adopt the techniques which we describe.

We only had two malignancies in our ten patients and it may be reasonably argued that a core biopsy would have avoided eight larger operations involving excision with potentially increased morbidity in these patients. However, in light of a radiology report suggesting a suspicious lesion, many surgeons and patients would elect to have definitive resective surgery in these circumstances. This is therefore debatable and would warrant more patient-doctor discussion.

\section{DECLARATIONS}

Ethical Approval Statement:. Ethical Approval was granted by the North-West Regional Health Authority.

\section{Conflict of Interest statement: None}

Informed Consent Statement: Signed, informed consent

was obtained from each patient participating in the study

Funding: None

Authors' Contributions: The house officers on the service (Brittney Lutchman, Adam Hanna, Jason Rattan, Johnathan Jarvis, Malini Ramnarine, Nicholas Babootee, Richard Spence, David Milne) - all reviewed the findings in each patient discussing as a group the patients' findings clinically and radiologically, critiquing the manuscript in each of the various stages of revision); Fidel Rampersad the sole interventional radiologist on the team conducting the radiologic procedures, preparing the radiology report and guiding the surgical approach, including the marking of the skin; Anna Rampersad obtaining and interpreting the signed consents, organizing the files and participating in the review meetings for discussing the manuscript; and Jameel Ali, the lead surgeon performing the surgical procedures, designing the protocol and critiquing the manuscript in its many versions.

\section{ACKNOWLEDGEMENTS:}

Participation by the Radiology Department and the attending medical staff is acknowledged.

\section{References:}

1. Taioli E, Attong-Rogers A, Layne P. Breast Cancer Survival in Women of African descent living in the US and in the Caribbean: effect of place of birth. Breast Cancer Res Treat. 2010; 122 (2): 515-530.

2. Donenberg $T$, Humeyun $A$, Royer R, Zhang $S$, Narod S, George S, Akbari A, Ali J, Hurley J. A Survey of BRCA1, BRCA2, and PALB2 mutations in women with Breast Cancer in Trinidad and Tobago. Breast Cancer Res Treat 2016: DOI 10.1007/s10549-3870-4.

3. American Cancer Society. Cancer Facts and Figures 2020. Atlanta. Ga: American Cancer Society; 2020.

4. Hayes MK. Update on Preoperative Breast Localization. Radiol Clin N Am 2017; 55: 591603.

5. Tang J, Xie XM, Wang X et al. Radiocolloid in combination with methylene dye localization rather than wire localization is a preferred procedure for excisional biopsy of non-palpable breast lesions. Ann Surg Oncol 2011;18 (1):109113.

6. Liu J, Guo W, Tong M. Intraoperative indocyanine green fluorescence guidance for excision of nonpalpable breast cancer. World J Surg Oncol 2016; 14 (1) 266-271.

7. Zografos GC, Doumitriou C, Lappas D, et al. Localization of non-palpable breast lesions using hook-wire combined with isosulfan blue dye. J Surg Oncol.2003:82 (1):73-74.

8. Sookar N,Narinesingh D, Ali J. Factors influencing Management strategies for Genetic Mutation Breast Cancer.J Oncol Ther:JONT-165DO1 10229011/2574-10X.0065.

9. Obenauer S, Hermann KP, Grabbe E. Applications and Literature Review of the BI-RADS classification. Eur Radiol 2005; 15: 1027-1036. 
